\title{
Efficacy and safety of geptanolimab (GB226) for relapsed or refractory peripheral T cell lymphoma: an open-label phase 2 study (Gxplore-002)
}

Yuankai Shi ${ }^{1 *} \mathbb{D}$, Jianqiu Wu ${ }^{2}$, Zhen Wang ${ }^{3}$, Liling Zhang ${ }^{4}$, Zhao Wang ${ }^{5}$, Mingzhi Zhang ${ }^{6}$, Hong Cen ${ }^{7}$, Zhigang Peng ${ }^{8}$, Yufu Li ${ }^{9}$, Lei Fan ${ }^{10}$, Ye Guo ${ }^{11}$, Liping Ma ${ }^{12}$, Jie Cui ${ }^{13}$, Yuhuan Gao ${ }^{14}$, Haiyan Yang ${ }^{15}$, Hongyu Zhang ${ }^{16}$, Lin Wang ${ }^{17}$, Weihua Zhang ${ }^{18}$, Huilai Zhang ${ }^{19}$, Liping Xie ${ }^{20}$, Ming Jiang ${ }^{21}$, Hui Zhou ${ }^{22}$, Yuerong Shuang ${ }^{23}$, Hang Su${ }^{24}$, Xiaoyan Ke ${ }^{25}$, Chuan Jin ${ }^{26}$, Xin Du ${ }^{27}$, Xin Du ${ }^{28}$, Li Liu ${ }^{29}$, Yaming Xi ${ }^{30}$, Zheng Ge ${ }^{31}$, Ru Feng ${ }^{32}$, Yang Zhang ${ }^{33}$, Shengyu Zhou ${ }^{1}$, Fan Xie ${ }^{34}$ and Qian Wang ${ }^{34}$

\begin{abstract}
Background: Peripheral T cell lymphoma (PTCL) is a rare disease and recent approved drugs for relapsed/refractory $(r / r)$ PTCL provided limited clinical benefit. We conducted this study to evaluate the efficacy and safety of geptanolimab (GB226), an anti-PD-1 antibody, in r/r PTCL patients.

Methods: We did this single-arm, multicenter phase 2 study across 41 sites in China. Eligible patients with $\mathrm{r} / \mathrm{r}$ PTCL received geptanolimab $3 \mathrm{mg} / \mathrm{kg}$ intravenously every 2 weeks until disease progression or intolerable toxicity. All patients who received at least one dose of geptanolimab and histological confirmed PTCL entered full analysis set (FAS). The primary endpoint was objective response rate (ORR) in FAS assessed by the independent radiological review committee (IRRC) per Lugano 2014 criteria.

Results: Between July 12, 2018, and August 15, 2019, 102 patients were enrolled and received at least one dose of geptanolimab. At the data cutoff date (August 15, 2020), the median follow-up was 4.06 (range 0.30-22.9) months. For 89 patients in FAS, 36 achieved objective response (40.4\%, 95\% Cl 30.2-51.4), of which 13 (14.6\%) were complete response and 23 (25.8\%) had partial response assessed by IRRC. The median duration of response (DOR) was 11.4 (95\% $\mathrm{Cl} 4.8$ to not reached) months per IRRC. Patients with PD-L1 expression $\geq 50 \%$ derived more benefit from geptanolimab treatment compared to $<50 \%$ ones (ORR, 53.3\% vs. 25.0\%, $p=0.013$; median PFS 6.2 vs. 1.5 months, $p=0.002$ ). Grade $\geq 3$ treatment-related adverse events occurred in 26 (25.5\%) patients, and the most commonly observed were lymphocyte count decreased $(n=4)$ and platelet count decreased $(n=3)$. Serious adverse events were observed in 45 (44.1\%) patients and 19 (18.6\%) were treatment related.
\end{abstract}

\footnotetext{
*Correspondence: syuankai@cicams.ac.cn

${ }^{1}$ Department of Medical Oncology, National Cancer Center/National

Clinical Research Center for Cancer/Cancer Hospital, Chinese Academy

of Medical Sciences \& Peking Union Medical College, Beijing Key

Laboratory of Clinical Study on Anticancer Molecular Targeted Drugs,

Beijing, China

Full list of author information is available at the end of the article
} original author(s) and the source, provide a link to the Creative Commons licence, and indicate if changes were made. The images or other third party material in this article are included in the article's Creative Commons licence, unless indicated otherwise in a credit line to the material. If material is not included in the article's Creative Commons licence and your intended use is not permitted by statutory regulation or exceeds the permitted use, you will need to obtain permission directly from the copyright holder. To view a copy of this licence, visit http://creativecommons.org/licenses/by/4.0/. The Creative Commons Public Domain Dedication waiver (http://creativeco mmons.org/publicdomain/zero/1.0/) applies to the data made available in this article, unless otherwise stated in a credit line to the data. 
Conclusions: In this study, geptanolimab showed promising activity and manageable safety profile in patients with r/r PTCL. Anti-PD-1 antibody could be a new treatment approach for this patient population.

Trial registration: This clinical trial was registered at the ClinicalTrials.gov (NCT03502629) on April 18, 2018.

Keywords: T cell lymphomas, PD-1 inhibitor, Immunotherapy

\section{Introduction}

According to the 2016 World Health Organization classification of lymphoid neoplasms [1], peripheral $\mathrm{T}$ cell lymphomas (PTCLs) were classified into 27 distinct subtypes. This heterogeneous disease entity accounts for around $25 \%$ of non-Hodgkin lymphoma (NHL) patients in China and about $10 \%$ in USA and Europe $[2,3]$. Due to its heterogeneity and rarity, most of the clinical studies conducted in PTCL were retrospective with a small sample size in which the treatment regimens largely followed B cell NHL. However, worse prognosis was associated with PTCLs when compared to B cell NHL as 5-year overall survival (OS) rate was only $32 \%$ in most common PTCL subtypes (PTCL not otherwise specified [PTCLNOS], angioimmunoblastic T cell lymphoma [AITL] and natural killer [NK]/T cell lymphoma) [3].

Anthracycline-containing regimens, for example CHOP (cyclophosphamide, doxorubicin, vincristine, prednisone) and CHOP plus etoposide (CHOEP), were commonly conducted in the first-line treatment of PTCL which have remained as the recommended approaches for most PTCL subtypes except for NK/T cell lymphoma in the first-line setting. For NK/T cell lymphoma, anthracycline-containing regimens usually provide poor clinical outcomes, whereas asparaginase-based chemotherapy, such as DDGP (dexamethasone, cisplatin, gemcitabline, and pegaspargase), can yield significant improvement in survival and better tolerability [4]. High-dose chemotherapy followed by autologous stem-cell transplantation (ASCT) has also been investigated [5-9]. For relapsed or refractory $(\mathrm{r} / \mathrm{r})$ PTCLs, novel agents like antifolate (pralatrexate) or histone deacetylase (HDAC) inhibitors (romidepsin, belinostat, vorinostat and chidamide) have shown an efficacy with objective response rates (ORRs) below 30\% [10-16].

Brentuximab vedotin, a CD30-directed antibody-drug conjugate, was recently reported for a promising efficacy in CD30-expressing systemic $r / r$ anaplastic large cell lymphoma (ALCL) with an ORR of $86 \%$ and duration of response (DOR) of 12.6 months [17]. Some evidence indicated that brentuximab vedotin could also bring clinical benefit to CD30-positive PTCL-NOS patients, not other subtypes of PTCL [18]. The unmet medical needs remain as new drugs for other targets to be explored and possibility to improve the treatment paradigm for $\mathrm{r} / \mathrm{r}$ PTCL.
In the past several years, immune checkpoint inhibitor (ICI) has revolutionized the clinical management of cancer [19]. The activity of anti-programmed cell death protein-1 (PD-1) has been observed in $\mathrm{r} / \mathrm{r}$ classic Hodgkin lymphoma and primary mediastinal large B cell lymphoma, in which programmed death-ligand 1 (PD-L1) upregulation is commonly seen [20-24]. Nevertheless, in $r / r$ diffuse large $B$ cell lymphoma (DLBCL), nivolumab showed limited efficacy with an independently assessed ORR of $10 \%$ and $3 \%$ in the ASCT-failed cohort and ASCT-ineligible cohort, respectively [25].

For $\mathrm{T}$ cell lymphoma, anti-PD-1 antibodies have shown modest efficacy in $r / r$ PTCL, but results were limited by a small sample size and concerns of rapid disease progression $[26,27]$. Thus, a better understanding of anti-PD-1 treatment for $\mathrm{r} / \mathrm{r}$ PTCL patients and identification of biomarkers for immunotherapy optimization are required.

Geptanolimab (GB226) is a recombinant anti-PD-1 humanized monoclonal antibody that selectively blocks the interaction of PD-1 with its ligands, manufactured by Genor Biopharma Co., Ltd (Shanghai, China). Based on the phase 1 study of geptanolimab in lymphoma and advanced solid tumors (data not published), $3 \mathrm{mg} / \mathrm{kg}$ every 2 weeks was selected as recommended. Herein, we present the results of this phase 2 study (Gxplore-002) to determine the efficacy and safety of geptanolimab in patients with $\mathrm{r} / \mathrm{r}$ PTCL, and assess the possible correlation between PD-L1 expression and its clinical outcome (NCT03502629).

\section{Methods}

\section{Patients}

Eligible individuals were patients aged 18 years and older with histologically confirmed PTCL, who had failed at least one prior systemic therapy. Additional criteria of enrolment included at least one bidimensionally measurable lesion according to investigator assessment as defined by Lugano criteria [28], an Eastern Cooperative Oncology Group (ECOG) performance status (PS) of 0-1, life expectancy of at least 3 months and at least 4 weeks after ASCT or major operation. Patients were also required to have adequate organ functions: absolute neutrophil count $\geq 1.0 \times 10^{9} / \mathrm{L}$, platelet count $\geq 75 \times 10^{9} / \mathrm{L}$, hemoglobin $\geq 80 \mathrm{~g} / \mathrm{L}$, total bilirubin $\leq 1.5 \times$ the upper limit of normal (ULN), alanine aminotransferase (ALT) and aspartate aminotransferase (AST) $\leq 2.5 \times \mathrm{ULN}$ 
(if liver involvements are present, ALT and AST up to $5 \times \mathrm{ULN}$ ), serum creatinine $\leq 1.5 \times \mathrm{ULN}$ or creatinine clearance $\geq 50 \mathrm{~mL} / \mathrm{min}$ (calculated by Cockcroft and Gault equation).

Patients were excluded if histologically classified as adult T cell leukemia/lymphoma (ATLL) or AITL; concomitant receipt of immunosuppressive therapy; prior exposure to any anti-PD-1/PD-L1 or anti-cytolytic T lymphocyte-associated antigen-4 (CTLA-4) antibody; treatment with systematic corticosteroids (>10 mg daily prednisone equivalent) within 2 weeks; active or history of autoimmune disease except for type I diabetes, controllable hypothyroidism with replacement treatment, controllable skin disorders without systematic treatment, celiac disease within control; human immunodeficiency virus (HIV) infection, anti-treponema pallidum antibody (TP-Ab) positive or active hepatitis B or C; use of any investigational agent, biologics or devices within 30 days (or at least five periods of half-life) before study treatment. Pathological diagnosis was done by study site pathologists for study enrolment, and patient eligibility was determined by site investigators. Central pathology review was conducted retrospectively upon patient consent.

The study was done in accordance with the Declaration of Helsinki and International Council for Harmonisation (ICH) guidelines for Good Clinical Practice. The study protocol is available in Additional file 1. The protocol, protocol amendments and patient informed consent were reviewed and approved by the relevant independent ethics committee at each participating study site prior to implementation. Written informed consent was obtained from all patients prior to enrolment.

\section{Study design, treatment and assessment}

We conducted this open-label, single-arm, phase 2 study across 41 sites in China to evaluate the activity and safety profile of geptanolimab in $\mathrm{r} / \mathrm{r}$ PTCL. Eligible patient received geptanolimab intravenously at a dose of $3 \mathrm{mg} / \mathrm{kg}$ every 2 weeks until disease progression, death, unacceptable toxicity, withdrawal of consent or end of the study (i.e., a maximum treatment duration of 2 years of the last subject, termination of treatment, consent withdrawal, lost to follow-up or death, whichever occurs first). Geptanolimab treatment was permitted to continue beyond the first assessment of progressive disease (PD) if the patient could benefit from the treatment with acceptable toxicity assessed by the investigator. Dose modification was not allowed. Patients with dose interruptions for more than 4 weeks should permanently discontinue the treatment.
Treatment response was assessed every 6 weeks until week 48 and every 12 weeks thereafter during the treatment phase. Treatment response was assessed using computed tomography (CT) or magnetic resonance imaging (MRI) and complementary information from positron emissions tomography and computed tomography (PET-CT). PET-CT was performed at baseline, week 13 and termination of treatment. All radiographic records of enrolled patients were provided to independent radiological review committee (IRRC). Efficacy was assessed based on individual best objective response by both investigators and IRRC according to the Lugano criteria [28]. Survival follow-up was done every 3 months until patient death, lost to follow-up or the end of the study.

\section{Outcomes}

The primary endpoint of this study was ORR, defined as the proportion of patients with a best overall response (including complete response [CR] and partial response [PR]) per the Lugano criteria and assessed by IRRC. The secondary outcomes included disease control rate (DCR), time to response (TTR), duration of response (DOR), PFS, OS, safety and immunogenicity of geptanolimab.

Safety was monitored for 30 days from the last dose of geptanolimab for all patients or before any anticancer therapy began. If no new anticancer treatment has commenced, the safety follow-up should be repeated as far as possible for 90 days after the last dose of geptanolimab. Adverse events (AEs) were assessed by National Cancer Institute Common Terminology Criteria for Adverse Event (NCI CTCAE), version 4.03 and classified as related, possibly related, unlikely related, unrelated and uncertainly related to treatment assessed by investigator. Treatment-related AEs (TRAEs) were those AEs labeled related, possibly related, or uncertainly related to study drug. As an ICI, geptanolimab could cause a spectrum of immune-related adverse events (irAEs), which is recognized and managed according to National Comprehensive Cancer Network guideline for Management of Immunotherapy-Related Toxicities [29].

\section{Statistical analysis}

To provide roughly $85 \%$ power to reject the null hypothesis that the true proportion of patients achieving an objective response is $15 \%$ or fewer and given a one-sided alpha of $2.5 \%, 73$ patients were required. Considering the possible drop-off rate of $15 \%$, at least 86 patients were to be recruited.

All patients receiving at least one dose of geptanolimab were included in the safety analysis set. Patients with retrospectively histological confirmation per central pathology review $(n=89)$ entered the full analysis set (FAS). 
Efficacy analysis was performed in FAS and per protocol set (PPS), assessed by IRRC and by investigators. ORR was the proportion of patients with CR or PR as the best overall response. DCR was the percentage of patients achieving either a CR or PR or SD. We summarized AEs as the proportion of the total number of patients receiving at least one dose of geptanolimab.

Exact binominal confidence intervals (CIs) were calculated for response outcomes. PFS (from first geptanolimab administration to progression or death, whichever occurs first), OS (from first geptanolimab administration to death), DOR (from the date of first documented response to the date of first documented disease progression or death from any cause) and TTR (time from first geptanolimab administration to first documentation of response) were assessed using the Kaplan-Meier method. All statistical analyses were performed by using SAS version 9.4.

This trial was registered at the ClinicalTrials.gov (NCT03502629).

\section{Biomarker assessment}

Immunogenicity was evaluated by the number of patients with antidrug antibody (ADA) positive. Serum samples were collected at baseline, week 1, 2, 6 and followed by every six weeks. An immunological ligand-binding assay (LBA) was developed and validated for the detection and quantitation of anti-geptanolimab antibodies in serum collected.

Archival tumor tissues were required from all patients prior to treatment. PD-L1 immunohistochemistry (IHC) was assessed on formalin-fixed paraffin-embedded (FFPE) tumor biopsy samples using Ventana PD-L1 (SP263; Roche) rabbit monoclonal primary antibody for exploratory analysis. Measure of PD-L1 expression built on the estimated percentage of PD-L1-stained cells. Genomic DNA was extracted from FFPE tumor samples using ReliaPrep FFPE gDNA Miniprep System (Promega) following the manufacturer's instructions. Microsatellite instability (MSI) analysis using MSI Analysis System version 1.2 (Promega) and tumor mutation burden (TMB) assessed with hybridization capture of exons from 440 cancer-related genes were performed (Details in Additional file 1).

\section{Results}

\section{Patients and treatment}

A total of 102 patients were recruited in this study at 41 sites in China from July 12, 2018, to August 15, 2019 (Additional file 1: Table S1). The average time for enrolled patients after the initial diagnosis of PTCL was 0.94 years (standard deviation: 1.53), and $58.8 \%$ of them had two or more prior treatments. At study entry, 84 (82.4\%) patients had advanced disease and 13 (12.7\%) patients had bone marrow involvement at baseline. All patients had received prior systematic treatment. For patients diagnosed with extranodal NK/T cell lymphoma, nasal type (ENKTL), 21 (91.3\%) out of 23 patients received prior asparaginase-based chemotherapy. For other subtypes of PTCL except for ENKTL, 77 (97.5\%) out of 79 patients were previously treated with anthracyclinecontaining chemotherapy. Twenty-four (23.5\%) patients had received chidamide; 7 (6.9\%) had undergone ASCT. Baseline characteristics of the 102 patients are shown in Table 1. Collectively, 89 patients with retrospectively histological confirmation per central pathology review were included in FAS (Additional file 1: Table S2). Of them, the most common histological subtypes were PTCL-NOS $(n=28$ [31.5\%]), ENKTL $(n=19$ [21.3\%]), anaplastic lymphoma kinase-negative anaplastic large cell lymphoma (ALCL ALK-, $n=13$ [14.6\%]) and anaplastic lymphoma kinase-positive anaplastic large cell lymphoma (ALCL ALK,$+ n=7$ [7.9\%]). At the cutoff date, 54 (52.9\%) deaths were recorded and $13(12.7 \%)$ patients were still under treatment (Fig. 2a). Disease progression was the most common reason for patient withdrawal, occurring in 50 (49.0\%) patients. Eleven (10.8\%) patients discontinued for TRAEs including two platelet count decreased, two autoimmune hemolytic anemia and two pneumonitis (Additional file 1: Table S3). All 102 patients were included in safety analysis and 89 with histological confirmation of PTCL were in FAS for efficacy analysis (Fig. 1).

\section{Efficacy}

As of August 15, 2020, the median follow-up was 4.06 (range 0.30-22.9) months. Efficacy data in the total enrolled population $(n=102)$ and FAS $(n=89)$ are presented in Table 2, with no significant difference observed between the two sets. Of FAS population, 36 of 89 (40.4\%, 95\% CI, $30.2 \%$ to $51.4 \%)$ patients achieved an overall response by IRRC with a median TTR of 1.4 (95\% CI, 1.4 to 1.5) months for responders; disease control was achieved in 53 (59.6\%; 95\% CI, 48.6\% to 69.8\%) patients. Assessed by investigators, 34 of 89 (38.2\%, 95\% CI, 28.1\% to $49.1 \%$ ) achieved an overall response (Table 2). The lower limit of the $95 \%$ CI (30.2\%) of ORR by IRRC was above the prespecified threshold of response. Best reductions from baseline in tumor burden are shown in Fig. 2b.

Among the 36 patients who responded to geptanolimab, 13 (14.6\%) patients achieved CR and 23 (25.8\%) achieved PR. Among them, the majority of patients had an ongoing response with a median DOR of 11.4 (95\% CI, 4.8 to not reached) months (Fig. 3a). The 12-month DOR was $48.5 \%$ (95\% CI, $29.6 \%$ to $65.0 \%$ ) (Table 2). Of FAS population, the median PFS was 2.7 (95\% CI, 2.2 to 4.2; Fig. 3b) months with a range of 0 to 21.2 months, and the 
Table 1 Patient baseline demographic and clinical characteristics $(N=102)$

\begin{tabular}{|c|c|}
\hline Patient characteristics & $N(\%)$ \\
\hline \multicolumn{2}{|l|}{ Age } \\
\hline Median age, years (range) & $52.5(18-78)$ \\
\hline$<65$ & $87(85.3)$ \\
\hline$\geq 65$ & $15(14.7)$ \\
\hline \multicolumn{2}{|l|}{ Gender } \\
\hline Male & $70(68.6)$ \\
\hline Female & $32(31.4)$ \\
\hline \multicolumn{2}{|l|}{ ECOG PS } \\
\hline 0 & $19(18.6)$ \\
\hline 1 & $83(81.4)$ \\
\hline \multicolumn{2}{|l|}{ Prior lines of systemic therapy } \\
\hline 1 & $42(41.2)$ \\
\hline 2 & $34(33.3)$ \\
\hline 3 or above & $26(25.5)$ \\
\hline \multicolumn{2}{|l|}{ Stage of disease $\mathrm{H}^{\#}$} \\
\hline$|-| \mid$ & $17(16.7)$ \\
\hline III-IV & $84(82.4)$ \\
\hline \multicolumn{2}{|l|}{ Pathological subtype } \\
\hline PTCL-NOS & $41(40.2)$ \\
\hline ENKTL & $23(22.5)$ \\
\hline ALCL ALK- & $12(11.8)$ \\
\hline ALCL ALK+ & $7(6.9)$ \\
\hline Other subtypes* & 19(18.6) \\
\hline \multicolumn{2}{|l|}{ Prior therapies } \\
\hline \multicolumn{2}{|l|}{ Multi-agent regimen } \\
\hline \multicolumn{2}{|l|}{ For ENKTL } \\
\hline Asparaginase-based chemotherapy $^{\dagger}$ & $21(91.3)$ \\
\hline \multicolumn{2}{|l|}{ For other subtypes of PTCL } \\
\hline Anthracycline-containing chemotherapy ${ }^{\ddagger}$ & $77(97.5)$ \\
\hline \multicolumn{2}{|l|}{ Single-agent regimen } \\
\hline Chidamide & $24(23.5)$ \\
\hline Gemcitabine & $55(53.9)$ \\
\hline Methotrexate & $10(9.8)$ \\
\hline Bortezomib & $1(1.0)$ \\
\hline Radiotherapy & $32(31.4)$ \\
\hline Autologous stem-cell transplantation & $7(6.9)$ \\
\hline
\end{tabular}

ENKTL, extranodal natural killer/T cell lymphoma, nasal type; ALCL, anaplastic large-cell lymphoma; ALK, anaplastic lymphoma kinase; $\mathrm{CHOP}$, cyclophosphamide, doxorubicin, vincristine, prednisone; ECOG, Eastern Cooperative Oncology Group; PS, performance status; NOS, not otherwise specified; PTCL, peripheral T cell lymphoma

\# Stage information of one case was not available

\$ PTCL was pathologically diagnosed by study site pathologist for study enrolment

* Other subtypes included: 11 cases of unclassifiable PTCL, 3 cases of type II enteropathy-associated T cell lymphoma (EATL, type II), 3 cases of mycosis fungoides (MF), 1 case of skin $\gamma \sigma$ cutaneous T cell lymphomas ( $\gamma \sigma \mathrm{CTCL})$, and 1 case of primary cutaneous $C D 4$ positive T cell lymphoma (CD4 + PCTCL)

+ The proportion (\%) was defined as the number of patients receiving asparaginase-based chemotherapy divided by the number of those diagnosed with ENKTL

₹ The proportion (\%) was defined as the number of patients receiving
Table 1 (continued)

anthracycline-containing chemotherapy divided by the number of those diagnosed with other subtypes of PTCL except for ENKTL

median OS was 14.6 (95\% CI, 9.6 to not reached) months, with a range of 0.7 to 22.9 months. No significant difference was observed between efficacy result per IRRC and investigator $(p=0.356)$.

Subgroup analysis was performed on FAS population showing consistent response across different age, gender, clinical stage, baseline bone marrow involvement and prior ASCT. Elevated lactate dehydrogenase (LDH) level was adversely correlated with patient response (ORR, $49.0 \%$ vs. $28.9 \%, p=0.081$; DCR, $72.5 \%$ vs. $42.1 \%$, $p=0.005$ ) and OS (not reached vs 9.5 months, $p=0.012$ ). Additionally, patients who were heavily treated $(\geq 2$ lines of systemic treatment) presented similar response (ORR $34.6 \%$ vs. $48.6 \%, p=0.197$ ) but shorter PFS ( 2.5 vs. 8.4 months, $p=0.001)$. Nevertheless, prior exposure to chidamide had no impact on patient response $(p=0.313)$. Among the 20 patients with previous failure of chidamide, response was observed in 6 patients $(30.0 \%$, 95\% CI 11.9 to 54.3) (Additional file 1: Table S4). Among patients in FAS, the highest IRRC-assessed ORR was observed in ENKTL reaching $63.2 \%$ (12/19), followed by $53.8 \%$ (7/13) in ALCL ALK-, 42.9\% (3/7) in ALCL ALK+ and $17.9 \%(5 / 28)$ in PTCL-NOS (Additional file 1: Table S5). Among the four patients classified as AITL according to the retrospectively central pathology review, two (50.0\%) achieved PR and two (50.0\%) achieved SD.

\section{Safety}

For 102 safety-evaluable patients, 94 (92.2\%) patients experienced at least one $\mathrm{AE}$ during the study and 35 (34.3\%) patients only had mild (grade 1-2) AEs. The most common AEs included white blood cell count decreased $(n=36,35.3 \%)$, anemia $(n=34,33.3 \%)$, fever $(n=27,26.5 \%)$, platelet count decreased $(n=24,23.5 \%)$, neutrophil count decreased $(n=20,19.6 \%)$, lymphocyte count decreased $(n=17,16.7 \%)$, hypokalemia $(n=16,15.7 \%)$, AST increased $(n=16,15.7 \%)$, cough $(n=16,15.7 \%)$, upper respiratory infection $(n=15$, $14.7 \%)$, anorexia $(n=15,14.7 \%)$, weight loss $(n=14$, $13.7 \%)$, ALT increased $(n=13,12.7 \%)$ and pruritus $(n=13,12.7 \%)$. According to investigator's assessment, TRAEs occurred in $80.4 \%$ of patients $(n=82$; Table 3$)$. TRAEs occurring in $\geq 10 \%$ were white blood cell count decreased $(n=20,19.6 \%)$, fever $(n=14,13.7 \%)$ and anemia $(n=13,12.7 \%)$. A total of $26(25.5 \%)$ patients occurred grade 3 or above TRAEs. The most commonly reported grade 3 or above TRAEs were lymphocyte count decreased $(n=4,3.9 \%)$, platelet count decreased $(n=3,2.9 \%)$, white blood cell count decreased $(n=2$, 


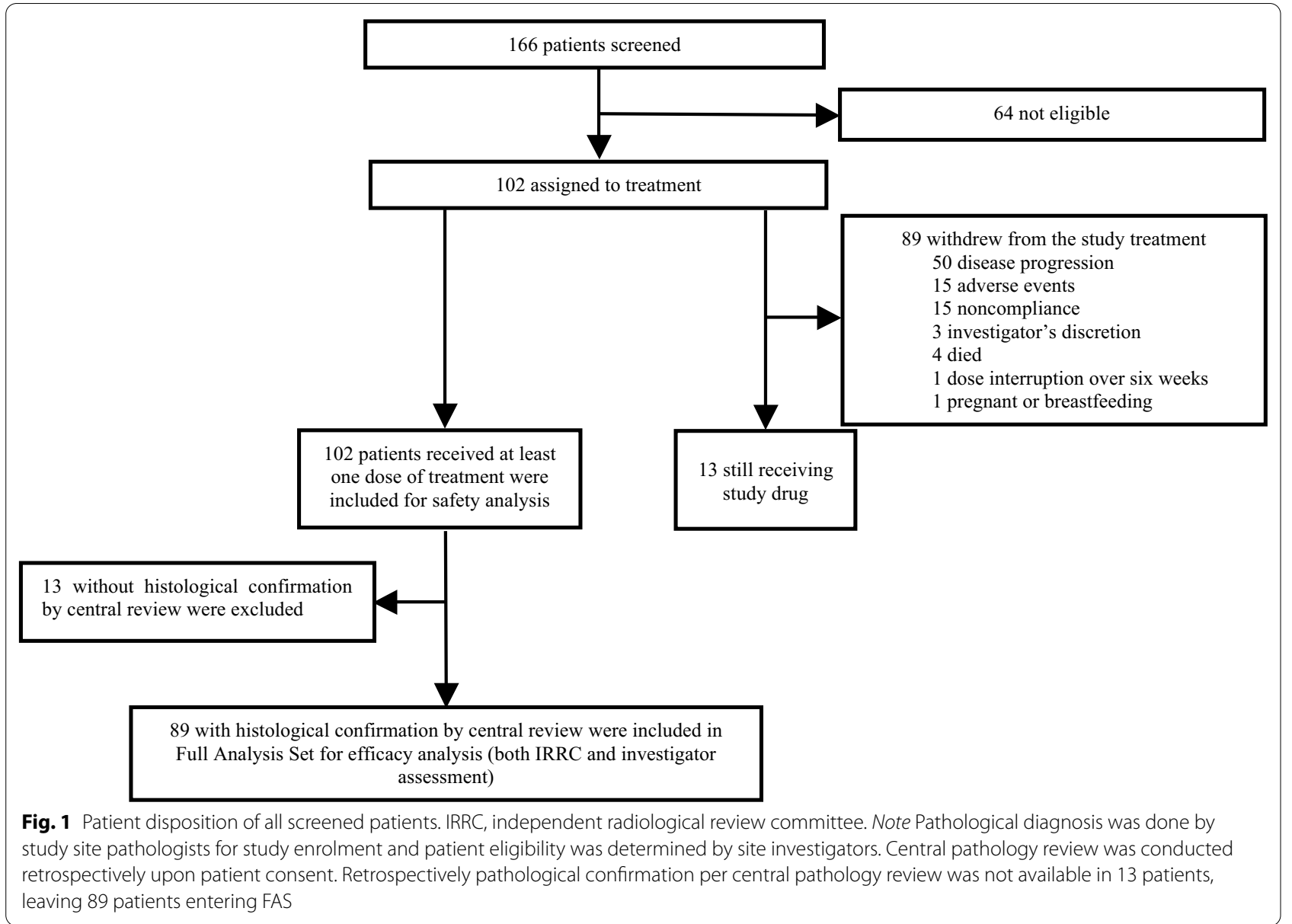

$2.0 \%)$, anemia $(n=2,2.0 \%)$, lung infection $(n=2,2.0 \%)$, upper respiratory infection $(n=2,2.0 \%)$, abnormal liver function $(n=2,2.0 \%)$, infection $(n=2,2.0 \%)$ and autoimmune hemolytic anemia $(n=2,2.0 \%)$. Severe adverse events (SAEs) were reported in $45(44.1 \%)$ patients, and 26 SAEs were judged to be treatment related in 19 (18.6\%) patients; the most common treatment-related SAE were platelet count decreased $(n=3,2.9 \%)$ and pneumonitis $(n=3,2.9 \%)$. There was no death attributed to the geptanolimab per investigator assessment.

irAE occurred in $36(35.3 \%)$ patients with 9.8\% $(n=10)$ of patients having grade 3 or above (Additional file 1: Table S6). The most common irAE was pruritus $(n=7,6.9 \%)$, followed by hypothyroidism $(n=4,3.9 \%)$, hyperthyroidism $(n=3,2.9 \%)$, tri-iodothyronine free decreased $(n=3,2.9 \%)$, tri-iodothyronine decreased $(n=3,2.9 \%)$ and thyroid-stimulating hormone increased $(n=3,2.9 \%)$. Of the 36 patients experiencing irAEs, the irAEs resolved without treatment or interruption of the geptanolimab in five patients. Twenty-two (21.6\%) patients received treatment for their irAEs, and $12(11.8 \%)$ of them received corticosteroids.

\section{Biomarker analysis}

Of 94 patients with ADA samples available, two (2.1\%) were tested positive for ADAs at baseline and one of them changed to be negative after geptanolimab administration. ADAs were detected in another four (4.3\%) patients during treatment course. Archival FFPE tissue samples were obtained for TMB $(n=59)$ and MSI testing $(n=67)$. Most patients $(n=65,97.0 \%)$ were microsatellite stable (MSS), and the median TMB was 1.2 (range 0-18.8) mutations/Mb.

Collectively, 91 patients had available tissue sample for PD-L1 testing. Of the 81 patients with available PD-L1 expression data in FAS, 80 (98.8\%) had PD-L1 expression of $1 \%$ or greater. More than half of patients $(45 / 81,55.6 \%)$ had a PD-L1 $\geq 50 \%$, and these patients derived more benefit from geptanolimab treatment compared to $<50 \%$ ones. Post hoc analysis showed ORRs were $53.3 \%$ 
Table 2 Efficacy of geptanolimab

\begin{tabular}{|c|c|c|c|c|}
\hline \multirow[t]{2}{*}{ Endpoint } & \multicolumn{2}{|l|}{ Full analysis set } & \multicolumn{2}{|l|}{ All patients enrolled } \\
\hline & $\begin{array}{l}\text { Investigator assessed } \\
(n=89)\end{array}$ & IRRC assessed $(n=89)$ & $\begin{array}{l}\text { Investigator assessed } \\
(n=102)\end{array}$ & IRRC assessed $(n=102)$ \\
\hline \multicolumn{5}{|l|}{ ORR } \\
\hline No. of patients & 34 & 36 & 37 & 39 \\
\hline$\%$ of patients $(95 \% \mathrm{Cl})$ & $38.2(28.1$ to 49.1$)$ & 40.4 (30.2 to 51.4$)$ & $36.3(27.0$ to 46.4$)$ & $38.2(28.8$ to 48.4$)$ \\
\hline \multicolumn{5}{|l|}{ DCR } \\
\hline No. of patients & 49 & 53 & 54 & 57 \\
\hline$\%$ of patients $(95 \% \mathrm{Cl})$ & 55.1 (44.1 to 65.6) & 59.6 (48.6 to 69.8$)$ & $52.9(42.8$ to 62.9$)$ & $55.9(45.7$ to 65.7$)$ \\
\hline \multicolumn{5}{|l|}{ Objective response, $n$ (\%) } \\
\hline$C R$ & $8(9.0)$ & $13(14.6)$ & $8(7.8)$ & $14(13.7)$ \\
\hline PR & $26(29.2)$ & $23(25.8)$ & $29(28.4)$ & $25(24.5)$ \\
\hline SD & $15(16.9)$ & $17(19.1)$ & $17(16.7)$ & $18(17.6)$ \\
\hline PD & $32(36.0)^{\#}$ & $28(31.5)$ & $37(36.3)^{\&}$ & $34(33.3)$ \\
\hline Not reported ${ }^{\S}$ & $8(9.0)$ & $8(9.0)$ & $11(10.8)$ & $11(10.8)$ \\
\hline \multicolumn{5}{|l|}{ Median DOR, months } \\
\hline Median $(95 \% \mathrm{Cl})$ & 4.0 (1.5 to NR) & 11.4 (4.8 to NR) & 4.2 (1.5 to NR) & 7.4 (5.1 to NR) \\
\hline$\%$ of patients $\geq 6$ months $(95 \% \mathrm{Cl})$ & 46.9 (29.1 to 62.8) & 60.5 (41.8 to 74.8$)$ & 48.6 (31.4 to 63.7$)$ & 60.9 (43.0 to 74.7$)$ \\
\hline$\%$ of patients $\geq 12$ months ( $95 \% \mathrm{Cl}$ ) & 46.9 (29.1 to 62.8$)$ & 48.5 (29.6 to 65.0$)$ & $40.8(23.9$ to 57.0$)$ & $43.8(26.3$ to 60.0$)$ \\
\hline
\end{tabular}

ORR, objective response rate; $D C R$, disease control rate; $C R$, complete response; $P R$, partial response; $S D$, stable disease; $P D$, progressive disease; IRRC, independent radiological review committee; $\mathrm{DOR}$, duration of response; $\mathrm{Cl}$, confidence interval; $\mathrm{NR}$, not reached

\# 21 patients had confirmed progressive disease; 11 patients with a first progressive disease and still under treatment

\& 23 patients had confirmed progressive disease; 14 patients with a first progressive disease and still under treatment

$\S$ Patients did not have post-baseline efficacy evaluation due to early termination or protocol violation

(24/45, 95\% CI $37.9 \%$ to $68.3 \%)$ in PD-L1 $\geq 50 \%$ patients and $25.0 \%(9 / 36,95 \%$ CI $12.1 \%$ to $42.2 \%)$ in $<50 \%$ cases $(p=0.013)$ (Fig. 4a). Additionally, PD-L1 $\geq 50 \%$ patients showed longer median PFS (6.2 months, 95\% CI, 2.7 to not reached vs. 1.5 months, $95 \%$ CI 1.3 to $2.7, p=0.002$; Fig. 4b) and OS (not reached, 95\% CI, 9.5 to not reached vs. 10.2 months, $95 \% \mathrm{CI}, 6.2$ to $18.5, p=0.043)$ than PD-L $1<50 \%$ cases.

\section{Discussion}

Studies in $\mathrm{r} / \mathrm{r}$ PTCL patients of second or later line treatment had a response rate ranging from 23 to $29 \%$ except brentuximab vedotin with a higher rate in CD30-positive PTCLs. In this study, treatment of geptanolimab demonstrated an IRRC assessed ORR of $40.4 \%$ in $\mathrm{r} / \mathrm{r}$ PTCL. A subgroup analysis showed direct association of response and survival with $\mathrm{PD}-\mathrm{L} 1 \geq 50 \%$ patients. To the best of our knowledge, this study represents the largest prospective clinical trial evaluating efficacy and safety of PD-1 inhibitor in PTCL patients.

PD-L1 expression has been approved in many indications as a determinative biomarker for conducting antiPD-1/PD-L1 treatment. Compared to DLBCL NOS, PD-L1 expression was higher in PTCLs [30], suggesting the possibility of adopting anti-PD-1 treatment in $\mathrm{T}$ and NK cell malignancies.

Preclinical data reported by Wartewig $\mathrm{T}$ et al. suggested that the oncogenic $\mathrm{T}$ cell signalling upregulated PD-1 expression while PD-1 suppressed oncogenic effector pathways. Additionally, their study found that ICIs could accelerate and/or reactivate $\mathrm{T}$ cell clones with oncogenically activated $\mathrm{T}$ cell receptor pathways, thus indicated the need for special consideration of using ICIs in patients with $\mathrm{T}$ cell non-Hodgkin lymphoma [31].

Some results of clinical studies indicated the concern of using PD-1 antibody in T cell lymphoma patients which might accelerate the tumor progression. A phase 2 study investigating nivolumab in ATLL, an aggressive mature T cell lymphoma characterized by human $\mathrm{T}$ cell leukemia virus type 1 (HTLV-1), discontinued after the first three patients showed a rapid progression by the first dose [32]. Further mechanism study suggested the detrimental effects derived from the correlation of PD-1 signaling and HTLV-1 basic zipper protein. In this study, we have excluded ATLL patients to avoid the possibility of rapid progression. In addition, a previous study of nivolumab in patients with $\mathrm{r} / \mathrm{r}$ PTCL, in which half of patients $(6 / 12)$ were AITL, reported hyperprogressive disease in four 
a
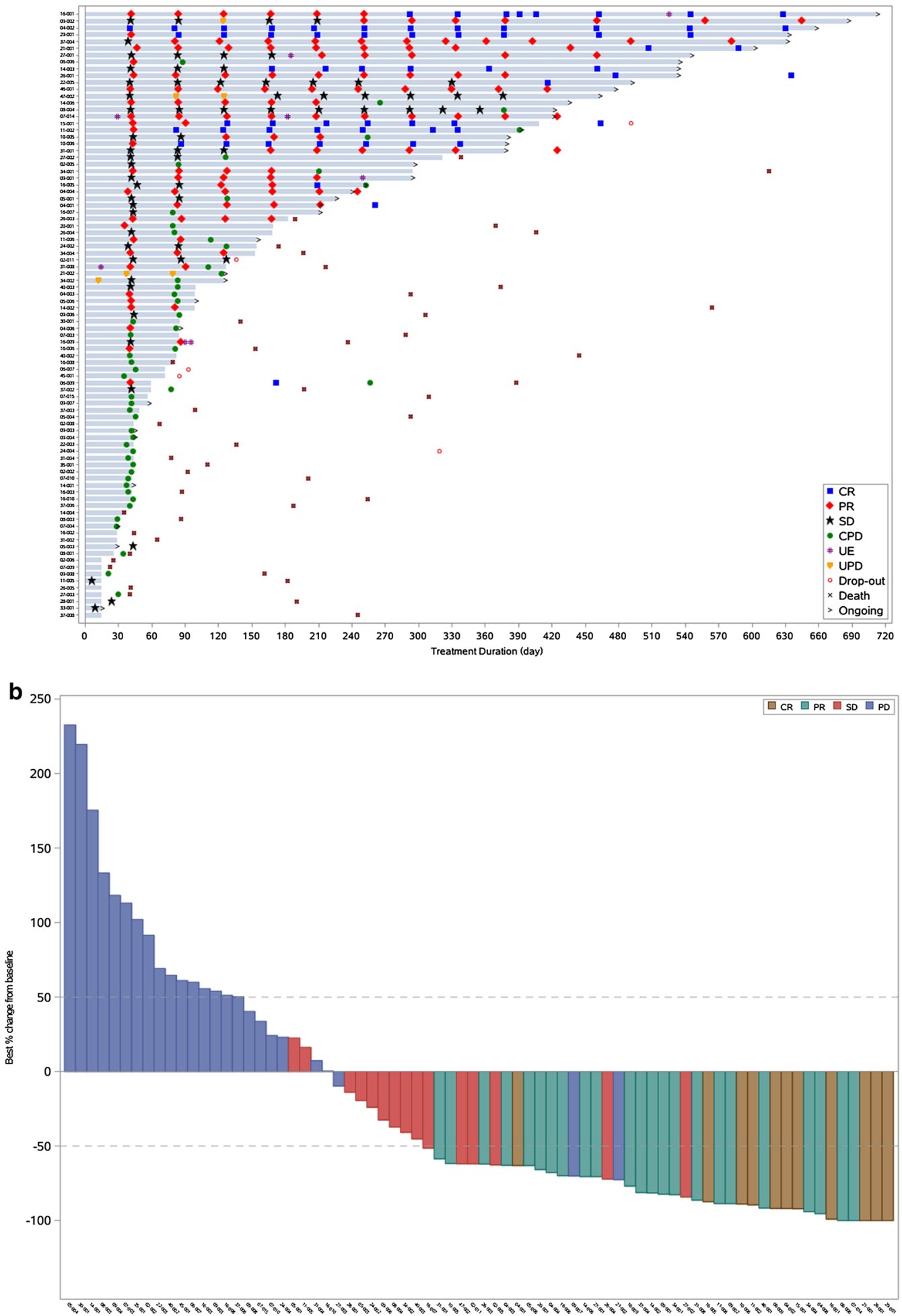

Fig. 2 a Duration of treatment for full analysis set population $(N=89)$. b Best percentage change from baseline in target lesion size for full analysis set population based on an independent radiological review committee assessment $(N=74)$. $C R$, complete response; $P R$, partial response; $S D$, stable disease; CPD, confirmed progressive disease; UE, unevaluable; UPD, unconfirmed progressive disease; PD, progressive disease. Note Out of 89 patients in the full analysis set, five patients did not have measurable target lesion at baseline per central review, eight patients withdrew before first response assessment and two patients had disease progression on the basis of physical examination by investigators without radiographic record 
a

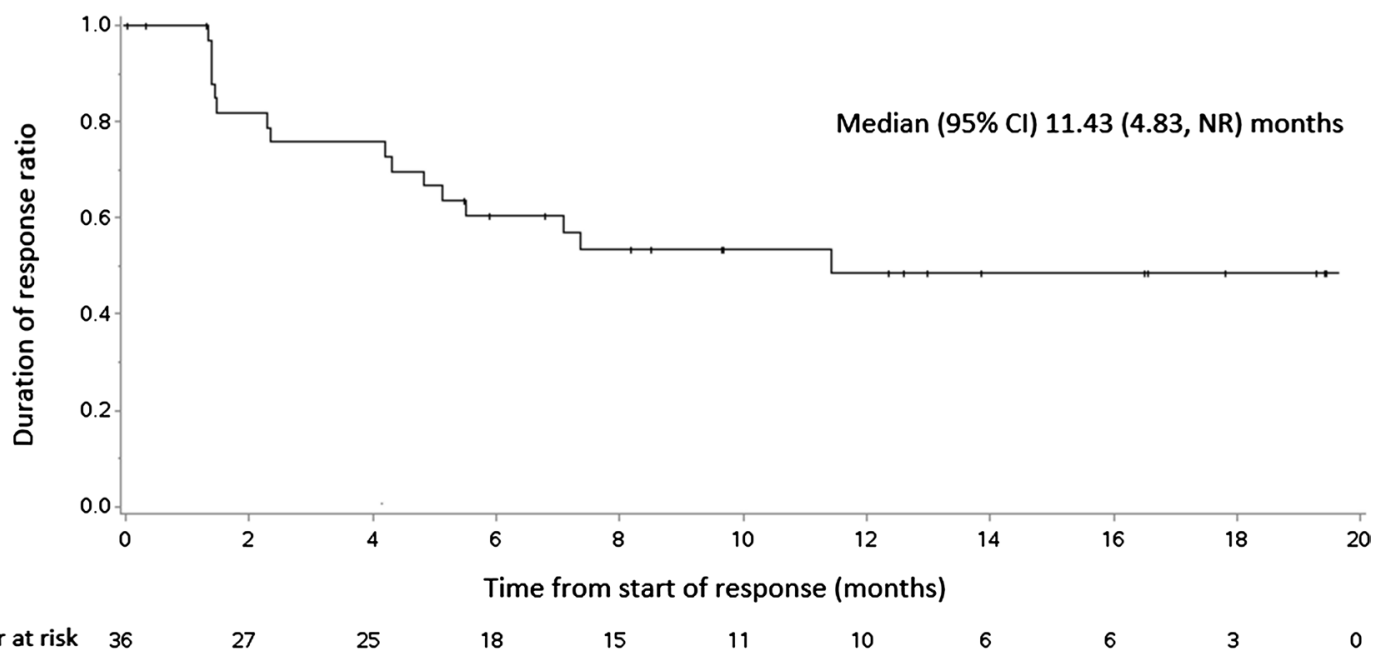

b

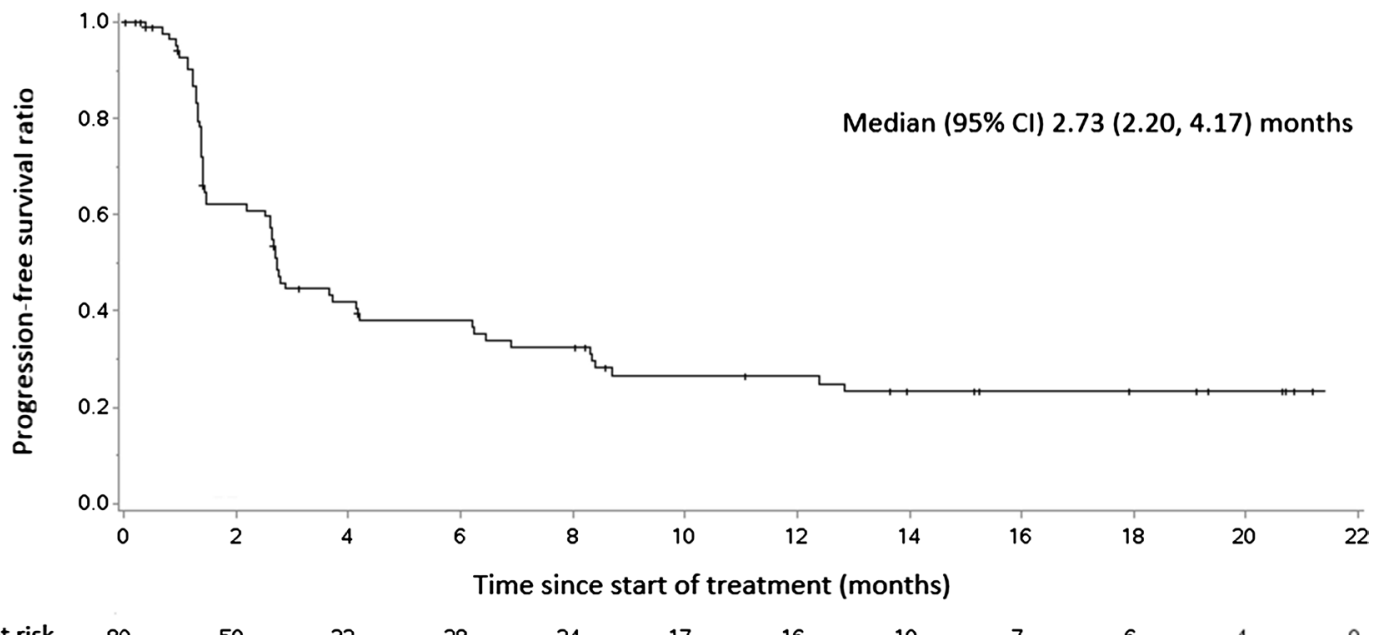

Fig. 3 a Kaplan-Meier estimated duration of response in full analysis set population based on an independent radiological review committee (IRRC) assessment of patients $(\mathrm{N}=36)$. b Kaplan-Meier estimated progression-free survival in full analysis set population based on an IRRC assessment $(\mathrm{N}=89)$. $\mathrm{NR}$, not reached; $\mathrm{Cl}$, confidence interval

patients [27]. Thus, we initially excluded AITL as well from enrolling in this study. Nevertheless, four patients were retrospectively confirmed AITL per central pathology review and all of them had disease control including $2 \mathrm{PR}$ and $2 \mathrm{SD}$. No hyperprogressive disease was observed in these four patients. Therefore, the efficacy and safety of anti-PD-1 treatment in AITL merit further investigations.

Other clinical data in PTCLs showed some activity. A phase 2 study of 23 enrolments with nivolumab treatment in $\mathrm{T}$ cell lymphoma represented an ORR of $17 \%$ in which all responders achieved PR. Two patients with
PTCLs had response durations of 10.6 and $78.6+$ weeks [33]. One more recent phase 2 trial of pembrolizumab monotherapy in $\mathrm{r} / \mathrm{r}$ mature $\mathrm{T}$ cell lymphoma showed an ORR of $33 \%$ (5 of 15 patients) but halted after interim futility analysis [26]. The ORR of this study was $40.4 \%$. Moreover, among the IRRC-assessed responders, the 12 -month DOR rate was $48.5 \%$, suggesting the survival benefit of geptanolimab for $r / r$ PTCLs. Two patients in this study recorded an increased tumor burden over $200 \%$ compared to baseline. The possibility of hyperprogression has been ruled out and determined as disease progression by the investigators. 
Table 3 Treatment-related adverse events

\begin{tabular}{|c|c|c|c|c|}
\hline Treatment-related adverse events* & Grade 1-2 & Grade 3 & Grade 4 & Grade 5 \\
\hline White blood cell count decreased & $18(17.6 \%)$ & $1(1.0 \%)$ & $1(1.0 \%)$ & 0 \\
\hline Fever & $13(12.7 \%)$ & $1(1.0 \%)$ & 0 & 0 \\
\hline Anemia & $11(10.8 \%)$ & $2(2.0 \%)$ & 0 & 0 \\
\hline Lymphocyte count decreased & $6(5.9 \%)$ & $3(2.9 \%)$ & $1(1.0 \%)$ & 0 \\
\hline Neutrophil count decreased & $9(8.8 \%)$ & 0 & $1(1.0 \%)$ & 0 \\
\hline Pruritus & $8(7.8 \%)$ & $1(1.0 \%)$ & 0 & 0 \\
\hline Platelet count decreased & $6(5.9 \%)$ & $1(1.0 \%)$ & $2(2.0 \%)$ & 0 \\
\hline Pneumonitis & $6(5.9 \%)$ & 0 & $1(1.0 \%)$ & 0 \\
\hline Rash & $4(3.9 \%)$ & $1(1.0 \%)$ & 0 & 0 \\
\hline Abnormal liver function & $3(2.9 \%)$ & $2(2.0 \%)$ & 0 & 0 \\
\hline Hypertension & $3(2.9 \%)$ & $1(1.0 \%)$ & 0 & 0 \\
\hline Upper respiratory tract infection & $1(1.0 \%)$ & $2(2.0 \%)$ & 0 & 0 \\
\hline Infusion related reaction & $2(2.0 \%)$ & $1(1.0 \%)$ & 0 & 0 \\
\hline Lung infection & 0 & $2(2.0 \%)$ & 0 & 0 \\
\hline Febrile neutropenia & 0 & 0 & $1(1.0 \%)$ & 0 \\
\hline Hyponatremia & 0 & $1(1.0 \%)$ & 0 & 0 \\
\hline Abdominal infection & 0 & $1(1.0 \%)$ & 0 & 0 \\
\hline Infection & 0 & $2(2.0 \%)$ & 0 & 0 \\
\hline Respiratory infection & 0 & $1(1.0 \%)$ & 0 & 0 \\
\hline Abdominal pain & 0 & $1(1.0 \%)$ & 0 & 0 \\
\hline Allergic reaction & 0 & $1(1.0 \%)$ & 0 & 0 \\
\hline Hyperuricemia & $3(2.9 \%)$ & 0 & $1(1.0 \%)$ & 0 \\
\hline Hypokalemia & $2(2.0 \%)$ & 0 & $1(1.0 \%)$ & 0 \\
\hline Heart failure & $1(1.0 \%)$ & 0 & $1(1.0 \%)$ & 0 \\
\hline Autoimmune hemolytic anemia & 0 & 0 & $2(2.0 \%)$ & 0 \\
\hline Autoimmune hepatitis & 0 & 0 & $1(1.0 \%)$ & 0 \\
\hline Volvulus ${ }^{\S}$ & 0 & 0 & 0 & $1(1.0 \%)$ \\
\hline Death ${ }^{\#}$ & 0 & 0 & 0 & $1(1.0 \%)$ \\
\hline Blood bilirubin increased & $1(1.0 \%)$ & 0 & $1(1.0 \%)$ & 0 \\
\hline Reticulocyte count increased & 0 & $1(1.0 \%)$ & 0 & 0 \\
\hline Lymphopenia & $1(1.0 \%)$ & $1(1.0 \%)$ & 0 & 0 \\
\hline Weight loss & $2(2.0 \%)$ & $1(1.0 \%)$ & 0 & 0 \\
\hline Bone marrow hypocellular & 0 & 0 & $1(1.0 \%)$ & 0 \\
\hline Neutropenia & 0 & $1(1.0 \%)$ & 0 & 0 \\
\hline
\end{tabular}

Data are number of patients (\%). This table included all grade treatment-related adverse events occurring in at least $10 \%$ of patients and all grade $3-5$ events

$\S$ One patient developed volvulus during treatment and resulted in death, which was deemed uncertainly related to geptanolimab treatment by investigator

\# The death was deemed uncertainly related to treatment

* Treatment-related adverse events were defined as an adverse event related, possibly related or uncertainly related to treatment, as assessed by the investigator

In this study, the $\mathrm{PD}-\mathrm{L} 1 \geq 50 \%$ rate was higher in patients with ENKTL $(78.9 \%, 15 / 19)$ and ALCL ALK+ $(71.4 \%, 5 / 7)$, followed by ALCL ALK- (38.5\%, 5/13) and PTCL-NOS (35.7\%, 10/28). For B cell lymphomas with PD-L1 gene alteration and upregulation, response rate was higher with anti-PD-1 treatment [2024]. We observed the correlation of PD-L1 expression with efficacy for PTCL patients in this study. Patients with PD-L1 expression of $50 \%$ or higher $(n=45)$ derived more benefit from geptanolimab treatment, showing an
ORR of $53.3 \%$ and a median PFS of 6.2 months. Additionally, response rate was higher for patients with ENKTL, ALCL ALK- or ALCL ALK+. The associations between PD-L1 expression, pathological subtypes and response to geptanolimab need further exploration in future clinical studies of PTCLs.

The safety profile of geptanolimab is similar to previous report of geptanolimab and other anti-PD-1 antibodies with hematologic disorders seeming to be more common [26, 27, 34]. Although TRAEs including fatigue, 

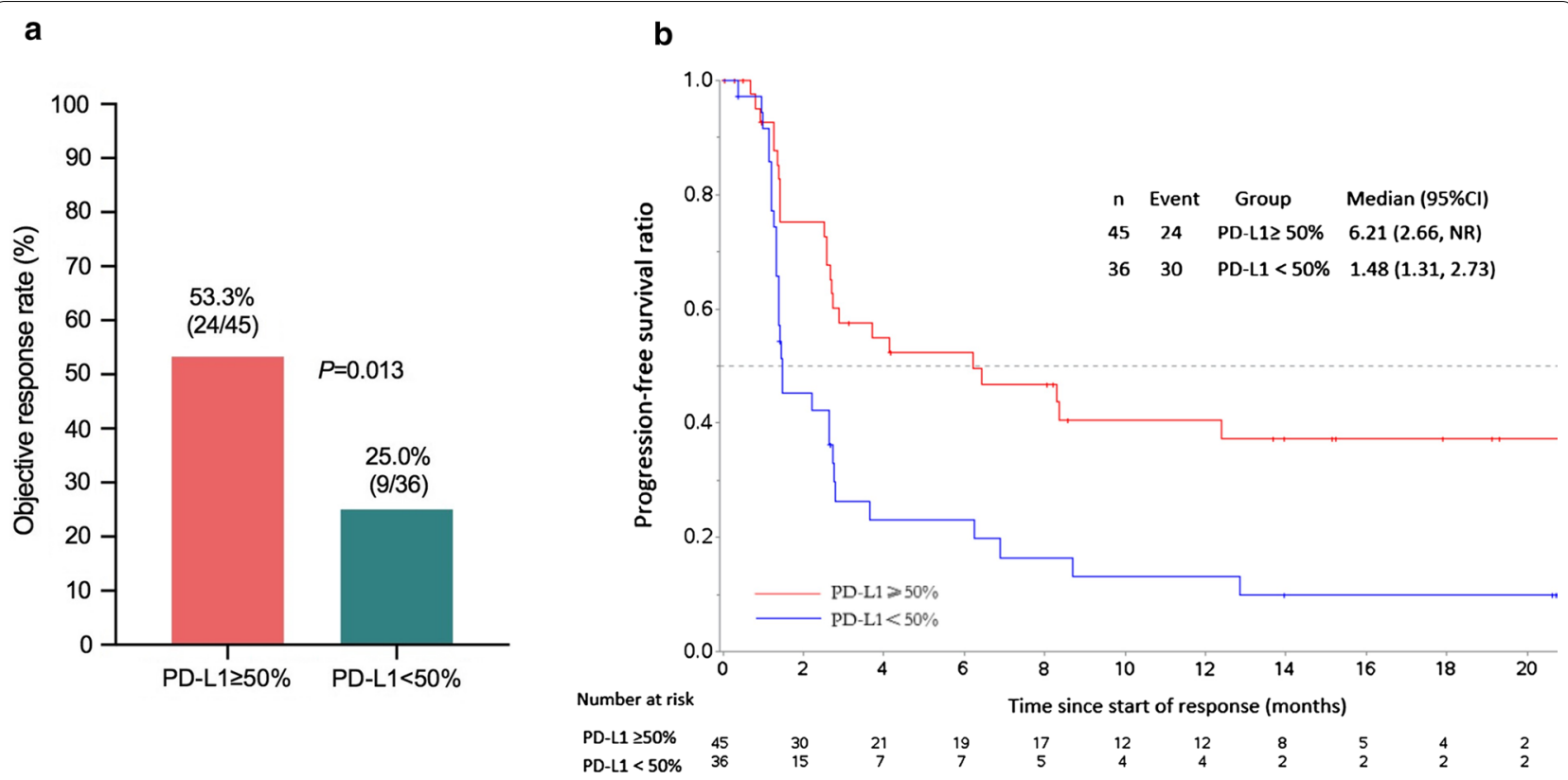

Fig. 4 a Post-hoc analysis of objective response rate and $\mathbf{b}$ Kaplan-Meier estimated of progression-free survival by PD-L1 expression based on an independent radiological review committee assessment. PD-L1, programmed death-ligand 1; Cl, confidence interval; NR, not reached. Note Responders were response-evaluable patients with PD-L1 assessed who achieved a complete or partial response as best overall response per 2014 Lugano lymphoma response criteria. PD-L1 expression was assessed with a cutoff of 50\%

gastrointestinal, skin disorders and pneumonitis were observed with anti-PD-1 treatment, the safety profile could vary in different malignancies [35, 36]. Patients with PTCLs are heterogenous and aggressive, likely to be treated with intensive chemotherapy previously; thus, patients with $r / r$ PTCL were more vulnerable to treatment. In this study, the most commonly reported grade 3 or 4 TRAEs were hematological, with lymphocyte count decreased and platelet count decreased reported in seven patients, and all cases are manageable.

Limitation of this study included absence of central pathology review in other 13 patients. In addition, this study is single arm, and the follow-up period was short. Therefore, a randomized control trial with longer followup period is warranted to further investigate the treatment in this population.

\section{Conclusions}

In conclusion, geptanolimab showed promising activity and manageable toxicity for patients with $\mathrm{r} / \mathrm{r}$ PTCL. Given this result, anti-PD-1 antibody could be a new treatment approach for patients with $\mathrm{r} / \mathrm{r}$ PTCL.

\section{Supplementary information}

The online version contains supplementary material available at https://doi. org/10.1186/s13045-021-01033-1.
Additional file 1: Supplemental Methods. Table S1 Study Site and Investigators. Table S2 Patient baseline demographic and clinical characteristics in full analysis set $(N=89)$. Table S3 Discontinuations due to Treatment-Related Adverse Events. Table $\mathbf{S} 4$ Subgroup analysis per independent radiological review committee $(n=89)$. Table S5 Efficacy of geptanolimab in pathological subtypes of PTCLs ${ }^{\&}$. Table $\mathbf{S 6} \mathbf{I m m u n e}$ related adverse events.

\section{Abbreviations}

PTCL: Peripheral T cell lymphoma; NHL: Non-Hodgkin lymphoma; PTCL-NOS: Peripheral T cell lymphoma, not otherwise specified; AITL: Angioimmunoblastic T cell lymphoma; NK: Natural killer; CHOP: Cyclophosphamide, doxorubicin, vincristine, prednisone; CHOEP: Cyclophosphamide, doxorubicin, vincristine, prednisone, etoposide; DDGP: Dexamethasone, cisplatin, gemcitabline, pegaspargase; ASCT: Autologous stem-cell transplantation; r/r: Relapsed or refractory; HDAC: Histone deacetylase; ALCL: Anaplastic large cell lymphoma; ORR: Objective response rate; DOR: Duration of response; ICI: Immune checkpoint inhibitor; PD-1: Programmed cell death protein-1; PD-L1: Programmed death-ligand 1; DLBCL: Diffuse large B cell lymphoma; ECOG: Eastern Cooperative Oncology Group; PS: Performance status; ULN: Upper limit of normal; ALT: Alanine aminotransferase; AST: Aspartate aminotransferase; ATLL: Adult T cell leukemia/lymphoma; CTLA-4: Cytolytic T lymphocyte-associated antigen-4; HIV: Human immunodeficiency virus; TP-Ab: Anti-treponema pallidum antibody; PD: Progressive disease; CT: Computed tomography; MRI: Magnetic resonance imaging; PET-CT: Positron emissions tomography and computed tomography; IRRC: Independent radiological review committee; CR: Complete response; PR: Partial response; DCR: Disease control rate; TTR: Time to response; PFS: Progression-free survival; OS: Overall survival; AE: Adverse event; TRAE: Treatment-related adverse event; irAE: Immune-related adverse event; FAS: Full analysis set; PPS: Per protocol set; Cl: Confidence interval; ADA: Antidrug antibody; LBA: Ligand-binding assay; IHC: Immunohistochemistry; FFPE: Formalin-fixed paraffin-embedded; MSI: Microsatellite instability; TMB: Tumor mutation burden; ENKTL: Extranodal natural killer/T cell lymphoma, nasal type; ALK: Anaplastic lymphoma kinase; LDH: Lactate dehydrogenase; HTLV-1: Human T cell leukemia virus type 1. 


\section{Acknowledgements}

This study was funded by Genor Biopharma Co., Ltd., China. Special thanks to the following key members: Dr. Joe Zhou, Dr. Yu Wang (consulting), Huiyang Cheng, Dr. Haige Shen (consulting), Shengbin Ren, Dr. Yue Kang, Dr. Tong Li, Dr. Chao Gao, Dr. Xifang Liu, Yi Zheng, Xuexiang Zhang, Ming Tong, Chunyan Yuan, Gaijing Xue, Cuncun Li, Jing Zhang, Jie Guo, Dr. Ziyi Kan, Zhen Gao, Jun Lin, Ke Tan, and Hongbin Yan. Authors also appreciate the contributions from all the investigators, the patients and their families. Finally, authors thank Shiyu Jiang (Department of Medical Oncology, Fudan University Shanghai Cancer Center, Shanghai Medical College, Fudan University) for her medical writing assistance, funded by Genor Biopharma Co., Ltd., China.

\section{Authors' contributions}

YKS was the leading principal investigator of this study, contributed to study conception and design, data analysis and interpretation, manuscript writing, revise and editing. YKS, JW, ZhenW, LZ, ZhaoW, MZ, HC, ZP, YL, LF, YGuo, LM, JC, YGao, HY, HYZ, LW, WZ, HLZ, LX, MJ, HZ, YRS, HS, XK, CJ, XD, XD, LL, YX, ZG, $R F, Y Z$ and $S Z$ were involved in patient recruitments and data acquisition. FX and QW contributed to data acquisition and data analysis. All authors had full access to all the data in this study and contributed to the writing of the report, reviewed it for intellectual content and approved the submitted version. All authors read and approved the final manuscript.

\section{Funding}

This study was funded by Genor Biopharma Co., Ltd., China. This study also received funding from China National Major Project for New Drug Innovation (Grant Nos. 2017ZX09304015) and Chinese Academy of Medical Sciences (CAMS) Innovation Fund for Medical Sciences (CIFMS) (Grant No. 2016-12M-1-001).

\section{Availability of data and materials}

The data that support the findings of this study are available from the corresponding author upon reasonable request.

\section{Ethics approval and consent to participate}

The study was done in accordance with the Declaration of Helsinki and ICH guidelines for Good Clinical Practice. The protocol, protocol amendments, and patient informed consent were reviewed and approved by the relevant independent ethics committee at each participating study site prior to implementation. Written informed consent was obtained from all patients prior to enrolment.

\section{Consent for publication}

Not applicable.

\section{Competing interests}

FX and QW are employees of Genor Biopharma Co., Ltd., China. The other authors declared no competing interests.

\section{Author details}

${ }^{1}$ Department of Medical Oncology, National Cancer Center/National Clinical Research Center for Cancer/Cancer Hospital, Chinese Academy of Medical Sciences \& Peking Union Medical College, Beijing Key Laboratory of Clinical Study on Anticancer Molecular Targeted Drugs, Beijing, China. ${ }^{2}$ Department of Oncology, Jiangsu Cancer Hospital, Nanjing, China. ${ }^{3}$ Department of Oncology, Linyi Cancer Hospital, Linyi, China. ${ }^{4}$ Department of Lymphoma, Cancer Center, Union Hospital, Tongji Medical College, Huazhong University of Science and Technology, Wuhan, China. ${ }^{5}$ Department of Hematology, Beijing Friendship Hospital, Capital Medical University, Beijing, China. ${ }^{6}$ Department of Oncology, The First Affiliated Hospital of Zhengzhou University, Zhengzhou, China. ${ }^{7}$ Department of Hematology, Lymphoma and Pediatric Oncology, Guangxi Medical University Affiliated Tumor Hospital and Oncology Medical College, Nanning, China. ${ }^{8}$ Department of Oncology, The First Affiliated Hospital of Guangxi Medical University, Nanning, China. ${ }^{9}$ Department of Hematology, Henan Cancer Hospital, Zhengzhou, China. ${ }^{10}$ Department of Hematology, Jiangsu Province Hospital, Nanjing, China. ${ }^{11}$ Department of Oncology, Shanghai East Hospital, Shanghai, China. ${ }^{12}$ Department of Hematology, Sun Yat-Sen Memorial Hospital Sun Yat-Sen University, Guangzhou, China. ${ }^{13}$ Department of Hematology, Gansu Provincial Cancer Hospital, Lanzhou, China. ${ }^{14}$ Department of Hematology, The Fourth Hospital of Hebei Medical University, Shijiazhuang, China. ${ }^{15}$ Department of Lymphoma, Zhejiang Cancer
Hospital, Hangzhou, China. ${ }^{16}$ Department of Oncology, The Fifth Affiliated Hospital Sun Yat-Sen University, Zhuhai, China. ${ }^{17}$ Department of Oncology, Hainan General Hospital, Haikou, China. ${ }^{18}$ Department of Hematology, The First Affiliated Hospital of Shanxi Medical University, Taiyuan, China. ${ }^{19}$ Department of Lymphoma, Tianjin Medical University Cancer Institute and Hospital, Tianjin, China. ${ }^{20}$ Department of Hematology, West China Hospital, Sichuan University, Chengdu, China. ${ }^{21}$ Department of Oncology, West China Hospital, Sichuan University, Chengdu, China. ${ }^{22}$ Department of Lymphoma and Hematology, Hunan Cancer Hospital, Changsha, China. ${ }^{23}$ Department of Lymphoma Hematology and Oncology, Jiangxi Cancer Hospital, Nanchang, China. ${ }^{24}$ Department of Lymphoma, The Fifth Medical Center of PLA General Hospital, Beijing, China. ${ }^{25}$ Department of Hematology, Peking University Third Hospital, Beijing, China. ${ }^{26}$ Department of Internal Medicine Section 4, Cancer Center of Guangzhou Medical University, Guangzhou, China. ${ }^{27}$ Department of Hematology, Guangdong Provincial People's Hospital, Guangdong Academy of Medical Sciences, Guangzhou, China. ${ }^{28}$ Department of Hematology, Shenzhen Second People's Hospital, Shenzhen, China. ${ }^{29}$ Department of Hematology, Tangdu Hospital of the Fourth Military Medical University, Xi'an, China. ${ }^{30}$ Department of Hematology, The First Hospital of Lanzhou University, Lanzhou, China. ${ }^{31}$ Department of Hematology, Zhongda Hospital Southeast University, Nanjing, China. ${ }^{32}$ Department of Hematology, Nanfang Hospital, Southern Medical University, Guangzhou, China. ${ }^{33}$ Department of Oncology, The Second Hospital of Dalian Medical University, Dalian, China. 34 Department of Medical Science, Genor Biopharma Co., Ltd., Shanghai, China.

Received: 12 November 2020 Accepted: 1 January 2021 Published online: 12 January 2021

\section{References}

1. Swerdlow SH, Campo E, Pileri SA, Harris NL, Stein H, Siebert R, et al. The 2016 revision of the World Health Organization classification of lymphoid neoplasms. Blood. 2016;127(20):2375-90.

2. Sun J, Yang Q, Lu Z, He M, Gao L, Zhu M, et al. Distribution of lymphoid neoplasms in China: analysis of 4,638 cases according to the World Health Organization classification. Am J Clin Pathol. 2012;138(3):429-34.

3. Vose J, Armitage J, Weisenburger D, International TCLP. International peripheral T-cell and natural killer/T-cell lymphoma study: pathology findings and clinical outcomes. J Clin Oncol. 2008;26(25):4124-30.

4. Li X, Cui Y, Sun Z, Zhang L, Li L, Wang X, et al. DDGP versus SMILE in newly diagnosed advanced natural Killer/T-Cell lymphoma: a randomized controlled, multicenter, open-label study in China. Clin Cancer Res. 2016;22(21):5223-8.

5. Feyler S, Prince HM, Pearce R, Towlson K, Smith IM, Schey S, et al. The role of high-dose therapy and stem cell rescue in the management of T-cell malignant lymphomas: a BSBMT and ABMTRR study. Bone Marrow Transplant. 2007;40(5):443-50.

6. Smith SM, Burns L, van Besien K, Lerademacher J, He W, Fenske TS, et al. Hematopoietic cell transplantation for systemic mature T-cell non-Hodgkin lymphoma. J Clin Oncol. 2013;31(25):3100-9.

7. Reimer P, Rudiger T, Geissinger E, Weissinger F, Nerl C, Schmitz N, et al. Autologous stem-cell transplantation as first-line therapy in peripheral T-cell lymphomas: results of a prospective multicenter study. J Clin Oncol. 2009;27(1):106-13.

8. d'Amore F, Relander T, Lauritzsen GF, Jantunen E, Hagberg H, Anderson H, et al. Up-front autologous stem-cell transplantation in peripheral T-cell lymphoma: NLG-T-01. J Clin Oncol. 2012;30(25):3093-9.

9. Gui L, Shi YK, He XH, Lei YH, Zhang HZ, Han XH, et al. High-dose therapy and autologous stem cell transplantation in peripheral T-cell lymphoma: treatment outcome and prognostic factor analysis. Int J Hematol. 2014;99(1):69-78.

10. O'Connor OA, Pro B, Pinter-Brown L, Bartlett N, Popplewell L, Coiffier $B$, et al. Pralatrexate in patients with relapsed or refractory peripheral T-cell lymphoma: results from the pivotal PROPEL study. J Clin Oncol. 2011;29(9):1182-9.

11. Piekarz RL, Frye R, Prince HM, Kirschbaum MK, Zain J, Allen SL, et al. Phase 2 trial of romidepsin in patients with peripheral T-cell lymphoma. Blood. 2011;117(22):5827-34. 
12. Coiffier B, Pro B, Prince HM, Foss F, Sokol L, Greenwood M, et al. Results from a pivotal, open-label, phase II study of romidepsin in relapsed or refractory peripheral T-cell lymphoma after prior systemic therapy. J Clin Oncol. 2012;30(6):631-6.

13. O'Connor OA, Horwitz S, Masszi T, Hoof AV, Brown P, Doorduijn J, et al. Belinostat in patients with relapsed or refractory peripheral T-cell lymphoma: results of the pivotal phase II BELIEF (CLN-19) study. J Clin Oncol. 2015;33(23):2492-9.

14. Olsen EA, Kim YH, Kuzel TM, Pacheco TR, Foss FM, Parker S, et al. Phase IIb multicenter trial of vorinostat in patients with persistent, progressive, or treatment refractory cutaneous T-cell lymphoma. J Clin Oncol. 2007;25(21):3109-15.

15. Shi Y, Dong M, Hong X, Zhang W, Feng J, Zhu J, et al. Results from a multicenter, open-label, pivotal phase II study of chidamide in relapsed or refractory peripheral T-cell lymphoma. Ann Oncol. 2015;26(8):1766-71.

16. Shi Y, Jia B, Xu W, Li W, Liu T, Liu P, et al. Chidamide in relapsed or refractory peripheral T cell lymphoma: a multicenter real-world study in China. J Hematol Oncol. 2017;10(1):69.

17. Pro B, Advani R, Brice P, Bartlett NL, Rosenblatt JD, Illidge T, et al. Five-year results of brentuximab vedotin in patients with relapsed or refractory systemic anaplastic large cell lymphoma. Blood. 2017;130(25):2709-17.

18. Horwitz SM, Advani RH, Bartlett NL, Jacobsen ED, Sharman JP, O'Connor OA, et al. Objective responses in relapsed T-cell lymphomas with singleagent brentuximab vedotin. Blood. 2014;123(20):3095-100.

19. Pardoll DM. The blockade of immune checkpoints in cancer immunotherapy. Nat Rev Cancer. 2012;12(4):252-64.

20. Chen R, Zinzani PL, Fanale MA, Armand P, Johnson NA, Brice P, et al. Phase II study of the efficacy and safety of pembrolizumab for relapsed/refractory classic hodgkin lymphoma. J Clin Oncol. 2017;35(19):2125-32.

21. Ramchandren R, Domingo-Domenech E, Rueda A, Trněný M, Feldman TA, Lee HJ, et al. Nivolumab for newly diagnosed advanced-stage classic Hodgkin lymphoma: safety and efficacy in the phase II CheckMate 205 study. J Clin Oncol. 2019;37(23):1997-2007.

22. Melani C, Major A, Schowinsky J, Roschewski M, Pittaluga S, Jaffe ES, et al. PD-1 Blockade in mediastinal gray-zone lymphoma. N Engl J Med. 2017;377(1):89-91.

23. Chapuy B, Stewart C, Dunford AJ, Kim J, Wienand K, Kamburov A, et al. Genomic analyses of PMBL reveal new drivers and mechanisms of sensitivity to PD-1 blockade. Blood. 2019;134(26):2369-82

24. Shi Y, Su H, Song Y, Jiang W, Sun X, Qian W, et al. Safety and activity of sintilimab in patients with relapsed or refractory classical Hodgkin lymphoma (ORIENT-1): a multicentre, single-arm, phase 2 trial. Lancet Haematol. 2019;6(1):e12-9.

25. Ansell SM, Minnema MC, Johnson P, Timmerman JM, Armand P, Shipp MA, et al. Nivolumab for Relapsed/Refractory Diffuse Large B-Cell Lymphoma in Patients Ineligible for or Having Failed Autologous Transplantation: A Single-Arm. Phase II Study J Clin Oncol. 2019;37(6):481-9.
26. Barta SK, Zain J, MacFarlane AWt, Smith SM, Ruan J, Fung HC, et al. Phase II study of the PD-1 inhibitor pembrolizumab for the treatment of relapsed or refractory mature T-cell lymphoma. Clin Lymphoma Myeloma Leuk 2019; 19(6): 356-64 e3.

27. Bennani NN, Pederson LD, Atherton P, Micallef I, Colgan JP, Thanarajasingam $\mathrm{G}$, et al. A phase II study of nivolumab in patients with relapsed or refractory peripheral T-cell lymphoma. Blood. 2019;134(Supplement_1):467.

28. Cheson BD, Fisher Rl, Barrington SF, Cavalli F, Schwartz LH, Zucca E, et al. Recommendations for initial evaluation, staging, and response assessment of Hodgkin and non-Hodgkin lymphoma: the Lugano classification. J Clin Oncol. 2014;32(27):3059-68.

29. Thompson JA, Schneider BJ, Brahmer J, Andrews S, Armand P, Bhatia S, et al. Management of immunotherapy-related toxicities, Version 1.2019. J Natl Compr Canc Netw. 2019;17(3):255-89.

30. Panjwani PK, Charu V, DeLisser M, Molina-Kirsch H, Natkunam Y, Zhao S. Programmed death-1 ligands PD-L1 and PD-L2 show distinctive and restricted patterns of expression in lymphoma subtypes. Hum Pathol. 2018;71:91-9.

31. Wartewig T, Kurgyis Z, Keppler S, Pechloff K, Hameister E, Öllinger R, et al. PD-1 is a haploinsufficient suppressor of T cell lymphomagenesis. Nature. 2017;552(7683):121-5.

32. Ratner $L$, Waldmann TA, Janakiram M, Brammer JE. Rapid progression of adult T-cell leukemia-lymphoma after PD-1 inhibitor therapy. N Engl J Med. 2018;378(20):1947-8.

33. Lesokhin AM, Ansell SM, Armand P, Scott EC, Halwani A, Gutierrez M, et al. Nivolumab in patients with relapsed or refractory hematologic malignancy: preliminary results of a phase lb study. J Clin Oncol. 2016;34(23):2698-704.

34. Shi Y, Cai Q, Jiang Y, Huang G, Bi M, Wang B, et al. Activity and Safety of Geptanolimab (GB226) for patients with unresectable, recurrent or metastatic alveolar soft part sarcoma: a phase 2 single-arm study. Clin Cancer Res. 2020;26(24):6445-52.

35. Nishino M, Giobbie-Hurder A, Hatabu H, Ramaiya NH, Hodi FS. Incidence of programmed cell death 1 inhibitor-related pneumonitis in patients with advanced cancer: a systematic review and meta-analysis. JAMA Oncol. 2016;2(12):1607-16.

36. Wang PF, Chen Y, Song SY, Wang TJ, Ji WJ, Li SW, et al. Immune-related adverse events associated with anti-PD-1/PD-L1 treatment for malignancies: a meta-analysis. Front Pharmacol. 2017;8:730.

\section{Publisher's Note}

Springer Nature remains neutral with regard to jurisdictional claims in published maps and institutional affiliations.

Ready to submit your research? Choose BMC and benefit from:

- fast, convenient online submission

- thorough peer review by experienced researchers in your field

- rapid publication on acceptance

- support for research data, including large and complex data types

- gold Open Access which fosters wider collaboration and increased citations

- maximum visibility for your research: over $100 \mathrm{M}$ website views per year

At BMC, research is always in progress.

Learn more biomedcentral.com/submissions 